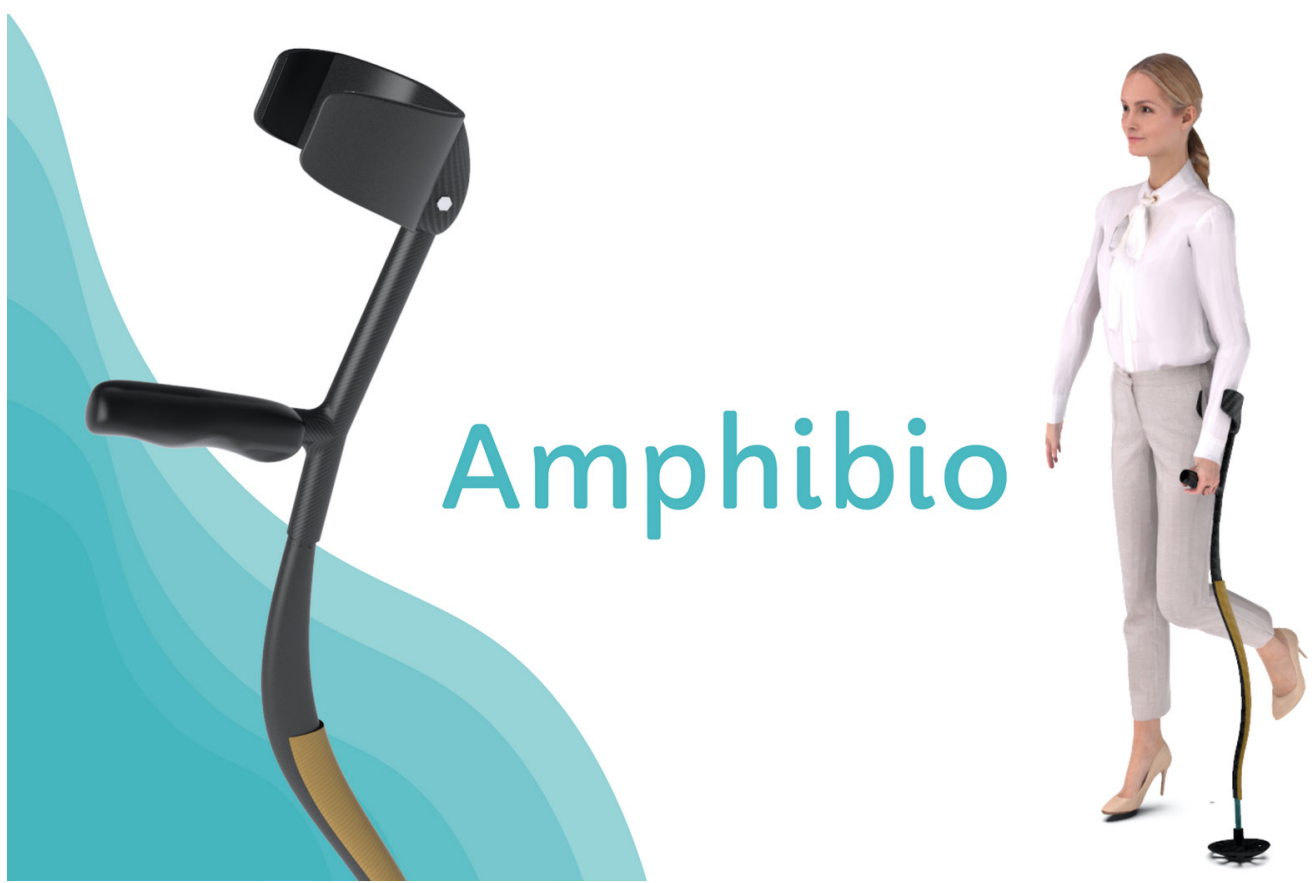

\title{
Optimización del diseño en manufactura y ensamblaje de un producto de ayuda técnica para personas con movilidad reducida en la playa
}

Design optimization in manufacturing and assembly of a technical assistance product for people with reduced mobility on the beach

Hilda Vásquez-Carvajal ${ }^{1}$, María Fernanda Arce-Murillo ${ }^{2}$, Tiffany Gamboa-Salas ${ }^{3}$

H. Vásquez-Carvajal, M.F. Arce-Murillo, T. Gamboa-Salas “Optimización del diseño en manufactura y ensamblaje de un producto de ayuda técnica para personas con movilidad reducida en la playa", IDI+, vol. 2, no 2, Ene., pp. 33-49, 2022.

doi) https: //doi.org/ 10.18845/ridip.v4i2.6073 Fecha de recepción: 28 de junio de 2021 Fecha de aprobación: 11 de noviembre de 2021

1. Hilda Vásquez-Carvajal

Estudiante de Ingeniería en Diseño Industrial Instituto Tecnológico de Costa Rica Cartago, Costa Rica hildarvc@estudiantec.cr (D) 0000-0003-2076-9207
2. María F. Arce-Murillo Estudiante de Ingeniería en Diseño Industrial Instituto Tecnológico de Costa Rica Cartago, Costa Rica mfer2017@estudiantec.cr (D) 0000-0003-2481-0289
3. Tiffany Gamboa-Salas Estudiante de Ingeniería en Diseño Industrial Instituto Tecnológico de Costa Rica Cartago, Costa Rica tiffanyG@estudiantec.cr (D) 0000-0002-8612-4682 


\section{Resumen}

Las playas han sido uno de los mayores atractivos turísticos de Costa Rica y el $76 \%$ de los turistas extranjeros las han frecuentado en su estadía en el país. De hecho, Costa Rica fue elegido el mejor destino a nivel mundial para la población jubilada. Pero, curiosamente, una de sus mayores problemáticas ha sido que muy pocas playas son accesibles y amigables para personas con discapacidad o adultos mayores. Actualmente, existen solo siete playas con rampas para sillas de ruedas o entradas accesibles en el país. Para solventar este problema, fueron diseñadas las muletas Amphibio, una ayuda técnica para que personas con movilidad inferior reducida puedan disfrutar de este ambiente. Se estudió y aplicó la metodología de Design for X y Design for Manufacture and Assembly (DFMA), con lo cual se logró optimizar el proceso de manufactura del producto y se concluyó con una nueva versión con mejoras significativas en su proceso de ensamble y fabricación. La implementación de estas metodologías en el desarrollo del producto logró generar un nuevo diseño tomando en cuenta no solo al usuario, sino también su fabricación y ensamblaje. Se observaron mejoras significativas en términos de cantidad de piezas, materiales principales, tipos de uniones y procesos de manufactura.

\section{Palabras Clave}

Manufactura; diseño industrial; ayuda técnica; producto inclusivo; playa

\section{Abstract}

The beaches are one of the most fantastic tourist attractions in Costa Rica, and 76\% of foreign tourists pass through our beaches during their stay in the country. In fact, Costa Rica was chosen as the best destination in the world for the retired population.

However, curiously, one of our beaches' most significant problems is that very few are accessible and friendly for people with disabilities or older adults. There are currently only seven beaches with wheelchair ramps or accessible entrances in the country. Amphibio crutches were designed to solve this problem, a technical aid so that people with reduced lower mobility can enjoy this environment. The Design for $X$ and Design for Manufacture and Assembly (DFMA) methodology was studied and applied to optimize the product's manufacturing process and conclude with a new version with significant improvements in its assembly and manufacturing process. By implementing these methodologies in the development of the product, a new design was generated. Through this, it considers the user and its manufacture and assembly. Significant improvements can be observed in terms of the number of parts, primary materials, types of joints, and manufacturing processes. 


\section{Keywords}

Manufacturing; industrial design; technical assistance; inclusive product; beach

\section{Introducción}

Alrededor de un 18,2 \% de la población costarricense tiene algún tipo de discapacidad [1]. Más allá, los adultos mayores en Costa Rica abarcan un 9,55 \% de la población y, según las estimaciones del Instituto Nacional de Estadística y Censos (INEC), se espera que este porcentaje se triplique en los próximos 40 años [2].

Por esta situación, Costa Rica está incursionando en la adaptabilidad de espacios recreativos para personas con discapacidad. El país tiene un proyecto con la Red Costarricense de Turismo Accesible, con el fin de ser el primer país en Centroamérica con playas accesibles. De aquí surge la necesidad de crear una ayuda técnica para personas con movilidad inferior reducida en la playa y colaborar con esta iniciativa para seguir fomentando la inclusividad.

Debido a lo anterior, se diseñan las muletas Amphibio. Mediante este producto, se pretende mejorar la accesibilidad de las playas de Costa Rica al ofrecerlas como un servicio para los turistas. El producto se diseña para poder utilizarse tanto dentro como fuera del agua, por lo tanto, tiene como requerimientos fundamentales poseer resistencia al agua salada y salitre, resistencia a los rayos UV, al calor y a la humedad; estar fabricado con un material que no genere fricción que pueda lastimar la piel de los usuarios; además, ser un material que pueda flotar y que sea ligero. Puede verse el modelo de las muletas en la figura 1.

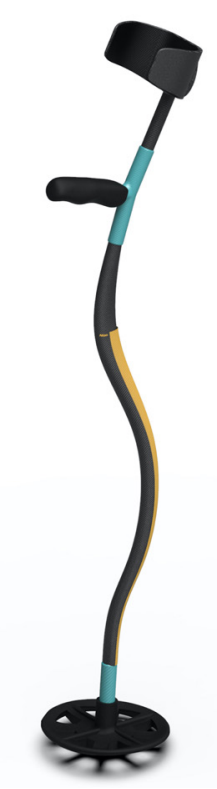

Fig. 1. Muletas Amphibio originales. 
Dentro de la configuración del producto inicial, se contempla crear diferentes tallas o módulos que pudieran variar dependiendo de la altura del codo, del largo del antebrazo y de la estatura del usuario. Esto con el fin de que sea un producto más accesible. Este detalle se puede ver en la figura 2.

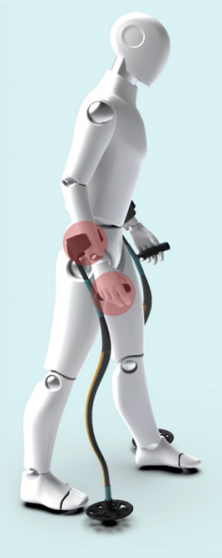

Talla Incorrecta
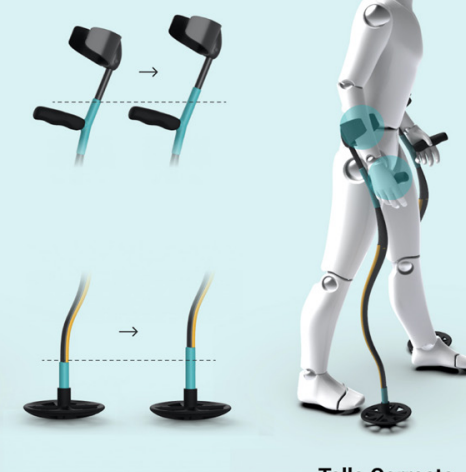

Fig. 2. Sistema de tallas.

Sin embargo, uno de los problemas identificados es la cantidad de piezas totales que posee el diseño, dado que se contempla un total de 11 piezas. Esto puede llevar a problemas de ensamblado, tiempos largos de fabricación y elevados costos de producción. Las partes del producto se pueden apreciar en la figura 3.

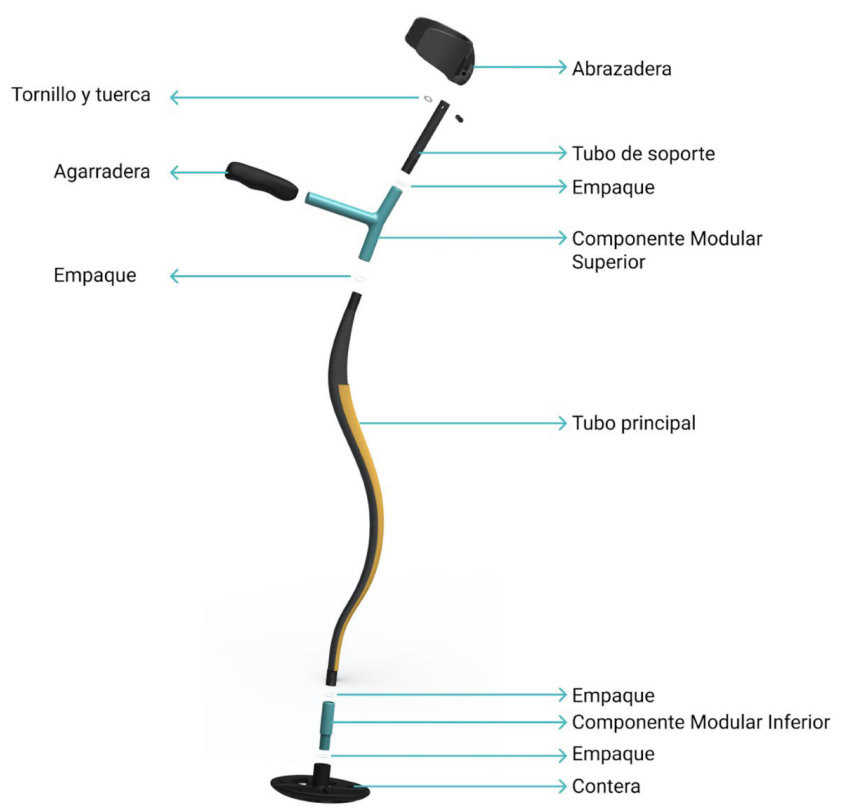

Fig. 3. Partes de las Muletas Amphibio iniciales. 
Otro de los problemas observados son las uniones del ensamblaje, las cuales son uniones roscadas (ver figura 4). Se llega a la conclusión de que estas uniones deben ser rediseñadas, debido a que la duración de ensamblaje, la posibilidad de fallas durante el armado y la hermeticidad del producto no se resolvían de la mejor manera, ya que existen mejores opciones para solucionar el problema.

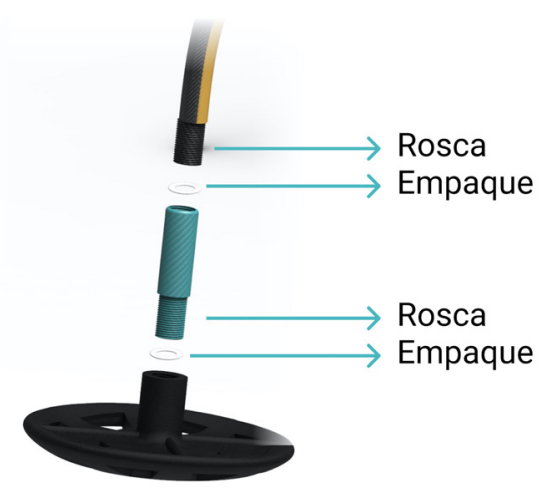

Figura 4. Unión roscada.

Por otro lado, el producto está fabricado con tres materiales, los cuales son fibra de carbono para la estructura principal, caucho natural para la contera y silicona como recubrimiento para los puntos de contacto con la piel del usuario. No obstante, el material de la fibra de carbono es conocido por tener un alto precio, lo cual es otro de los problemas por resolver, ya que afecta la rentabilidad del producto.

La consideración de los problemas de fabricación y ensamblaje en la etapa de diseño del producto es, por lo tanto, la forma más rentable disponible para reducir los costos de ensamblaje y aumentar la productividad [3]. Por lo que este artículo se basa en el planteamiento para mejorar la manufactura y el ensamble de este producto, tomando en cuenta el diseño explicado previamente como base para la investigación.

\section{Metodología}

\section{Design for Manufacture and Assembly (DFMA)}

Una vez que se obtuvo el diseño del producto, se procedió con el análisis de su manufactura y ensamble, donde se aplicó DFMA, la cual es una metodología de ingeniería que se centra en simplificar el diseño de un producto para mejorar la facilidad de fabricación y la eficiencia del montaje [4]. Al ser aplicada, se aspira a integrar y coordinar los procesos de diseño y fabricación desde el inicio de las etapas de diseño y desarrollo, así como lograr la congruencia entre los procesos de diseño y manufactura. Más que pasos o etapas, el DFMA posee lineamientos que guían al diseñador durante el proceso. 
Para efectos del desarrollo del trabajo, se tomaron en cuenta lineamientos como reducir al mínimo el número de partes y componentes de la configuración del producto, reducir al mínimo la cantidad de números de piezas presentes, conocer los requisitos ergonómicos y accesibles [5]. Asimismo, el lineamiento de facilitar el armado por medio de ensambles simples a prueba de fallos y errores del usuario; a su vez, los ensambles contribuyen a un fácil mantenimiento [5]. Se contempló el lineamiento de analizar la estructura del producto de acuerdo con las tecnologías disponibles para su manufactura [5].

\section{Design for X (DFX)}

La metodología DFX es el principal pilar de la ingeniería concurrente. Consiste en métodos, directrices y estándares para crear productos de mejor calidad en la fase de diseño conceptual. Bajo el nombre de "Diseño para la excelencia", es adoptada por las empresas como parte integral del proceso de desarrollo de productos de mayor calidad, menor costo del producto y ciclos de desarrollo de productos más cortos [6]; gracias a la modularidad en el diseño de productos [7], diseño para el desmontaje (DFD) [8], diseño para la logística de materiales (DFML) [9], diseño para el medio ambiente (DFE) [10], diseño para el servicio (DFS) [11], diseño para mantenibilidad (DFM) [12], entre otras metodologías que componen al DFX.

Tomando en cuenta las metodologías anteriormente descritas, se realizaron las siguientes fases:

\section{Fase 1: Análisis del producto}

- Se realiza un análisis general del producto inicial. Este puede ser de tipo configuracional, de utilidad, funcional, de materiales, perceptual, de sus procesos de manufactura, tecnologías necesarias para poder materializar el producto, etc. Esto con el fin de establecer requisitos y requerimientos de su forma y configuración.

- Se detalla la configuración de cada pieza del producto. Para esto, se realiza el modelado del producto tomando en cuenta los detalles constructivos, de montaje (uniones, articulaciones, acoplamientos, cierres, etc.) y los materiales propuestos para cada pieza.

- Se enlistan los componentes de mercado (estandarizados y normalizados) y establecen los materiales idóneos en conjunto con las tecnologías pertinentes.

\section{Fase 2: Procesos de manufactura para piezas y montaje}

- Se analiza la estructura y arquitectura del producto. Esto con el objetivo de establecer la interacción entre partes e interfaces de cada pieza.

- Se analiza el diseño del producto versus el proceso de manufactura empleado, utilizando la metodología del Diseño para Manufactura y Ensamble (DFMA). 


\section{Fase 3: DFMA}

- Se establecen mejoras en el diseño y el proceso de manufactura. Posteriormente, se implementan los cambios necesarios de acuerdo con el DFMA.

\section{Resultados}

Siguiendo la metodología descrita anteriormente, se logra desarrollar cambios que mejoran el producto diseñado, tanto en su configuración como en su proceso de manufactura, basado en las metodologías implementadas, principalmente DFMA.

Dentro de los resultados obtenidos, existen tres que hacen el producto más eficiente tanto a nivel de manufactura como de uso. El primero de ellos es el tipo de materiales que se determinó usar, los cuales fueron seleccionados por su alta resistencia en las condiciones presentes en las playas y su durabilidad al ser utilizado. El segundo resultado importante fue la cantidad de piezas y partes utilizadas en la configuración de las muletas. El diseño inicial estaba compuesto por 11 piezas, sin contabilizar los componentes estandarizados, donde estaba la abrazadera, el tubo de soporte, la agarradera, componente modular superior (3), el tubo principal, componente modular inferior (3) y la contera.

Al aplicar las metodologías, se logra reducir la cantidad a cinco piezas, donde cada una de ellas presenta una mejora implementada. En la figura 5, se observa un resumen de los principales cambios a nivel de ensambles. De este modo, se reduce el tiempo necesario para su ensamblaje y facilita comprender su uso.

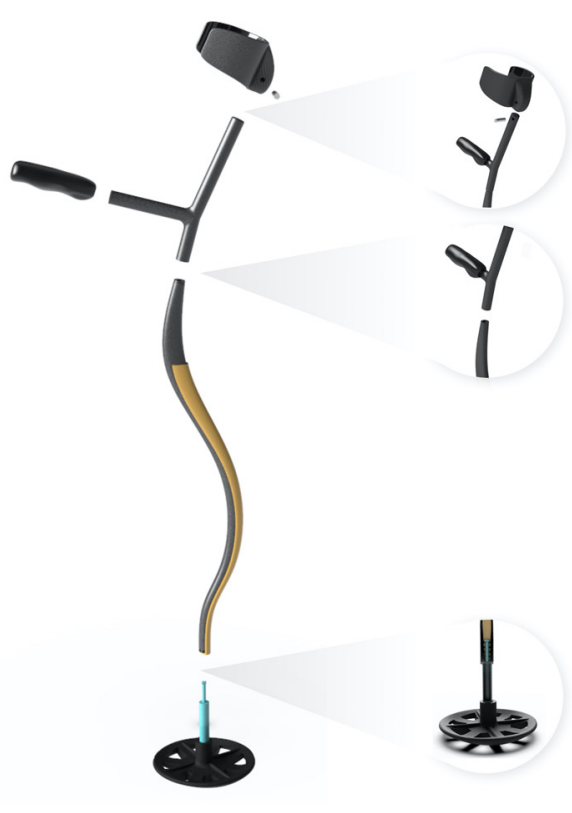

Fig. 5. Resumen de nuevos ensambles. 
La abrazadera: esta pieza se mantiene con la forma definida originalmente, sin embargo, al ser ensamblada a las demás, se realiza con el uso de un pasador que permite que esta abrazadera sea articulada y pueda moverse angularmente, para adaptarse a la altura del brazo del usuario, promoviendo la modularidad del producto. Se observa con más detalle en la figura 6

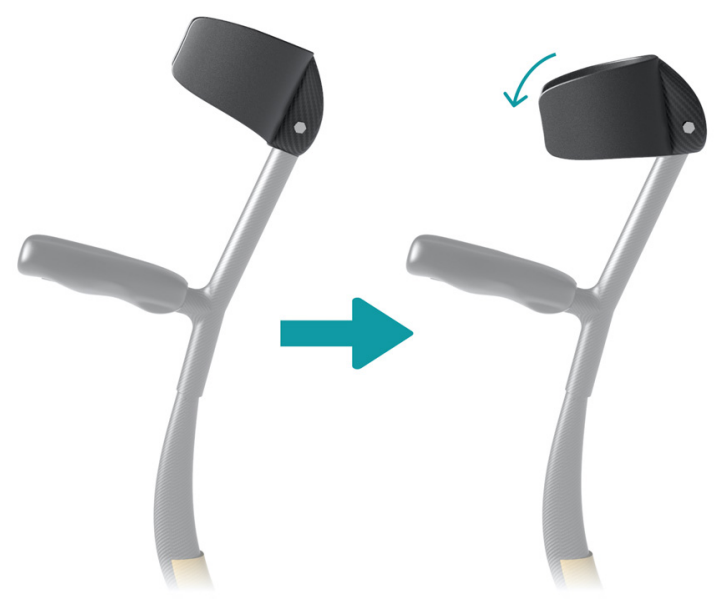

Fig. 6. Abrazadera articulada.

El tubo de soporte: el diseño inicial presentaba dos tubos que componían el tubo de soporte y el de la agarradera, esto porque uno de ellos correspondía a la pieza modular con diferentes dimensiones, donde, al ser analizado, se determina que la diferencia en las dimensiones eran mínimas, por lo que se procedió a eliminar dicha pieza, haciendo una única pieza, lo que disminuye la cantidad y acelera el proceso de producción. Esto se puede observar con más detalle en la figura 7.

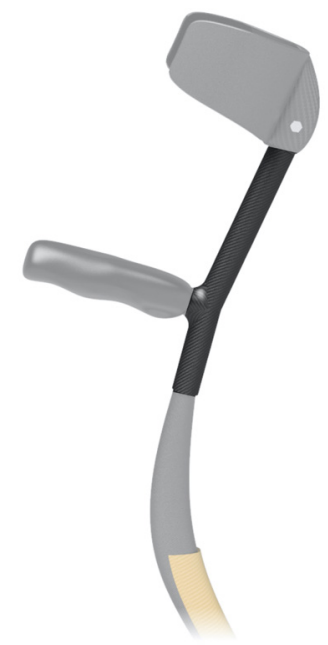

Fig. 7. Tubo de soporte unificado. 
La agarradera: se realiza un recubrimiento de silicona para la agarradera, donde existe un alto contacto por parte del usuario, con el fin de brindarle mayor comodidad al utilizar el producto, y evitar que tenga una posibilidad de lastimar sus manos. Se puede ver el detalle en la figura 8.

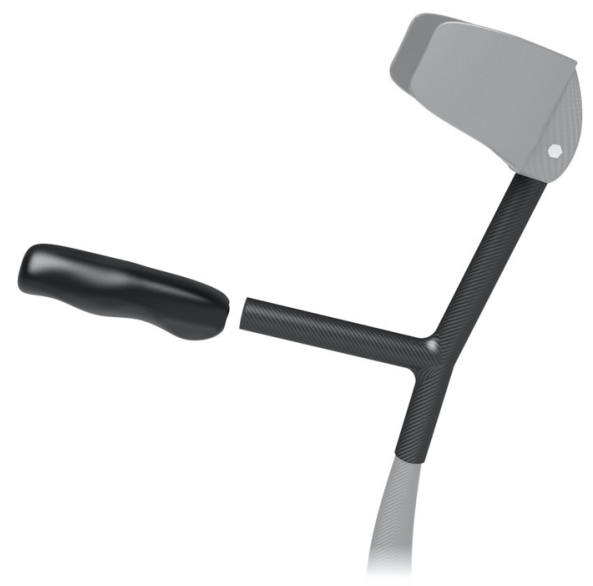

Fig. 8. Recubrimiento de la agarradera.

El tubo principal: esta pieza presenta alta complejidad en su manufactura, por lo que se determina que sea una pieza aparte de las demás, con el propósito de que su morfología se respete al manufacturarse, ya que permite distribuir eficientemente las fuerzas implementadas en la muleta y, en caso de que esta se dañe, pueda ser fácilmente reemplazada sin desechar toda la muleta, reduciendo el uso de material en la producción. Esta forma se puede observar en la figura 9.

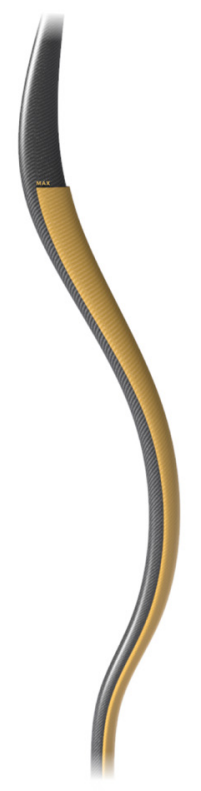

Fig. 9. Tubo principal. 
La contera regulable: esta pieza está realizada por un subensamble, donde, en el tubo que permite regular la altura de la muleta y brinda la modularidad buscada en las muletas, es insertada la contera. Se puede observar en la figura 10.

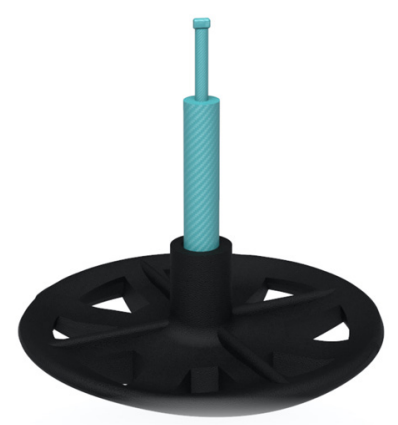

Fig. 10. Contera regulable.

El último aspecto mejorado en el producto diseñado fueron sus tipos de uniones y ensambles. El diseño inicial contaba con uniones tipo rosca para cada pieza, sin embargo, siguiendo las pautas del DFMA, se determina que esto debe ser cambiado y se realiza un análisis de tipos de uniones y métodos de ensamblaje. Como se observa en la figura 11, el ensamble de la abrazadera al tubo de soporte se realiza por medio de un pasador (componente estandarizado) que permite su articulación.

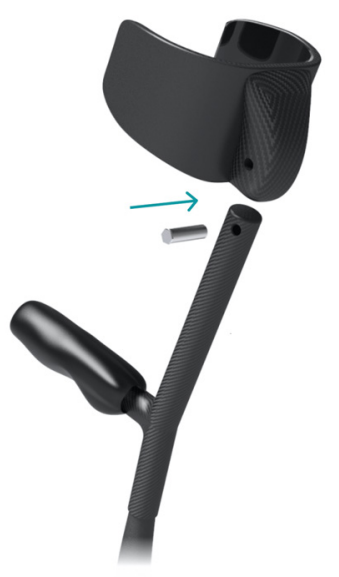

Fig. 11. Unión de pasador.

La unión entre el tubo de soporte y el tubo principal se realiza por medio de un ensamble por presión (como se observa en la figura 12), donde el tubo principal es insertado en el interior del otro, en el que se fija su posición por la fricción del material. El tubo regulable es ensamblado en la contera cuando esta se encuentra en su proceso de manufactura, ya que este se inserta en el molde donde el caucho se conforma. 


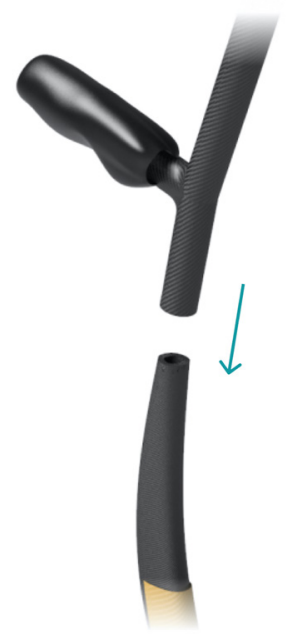

Fig. 12. Unión por presión.

Como se observa en la figura 13 , se implementa una unión entre el tubo principal y el tubo regulable que permite cambiar la altura de la muleta, por medio de gradientes internos, donde el tubo inferior encaja dentro de las paredes internas del otro tubo, en el que es girado y se fija para poder ser utilizado. De esta manera, los ensambles implementados en el nuevo diseño permiten agilizar el proceso de ensamblado, reduciendo el tiempo necesario para ello

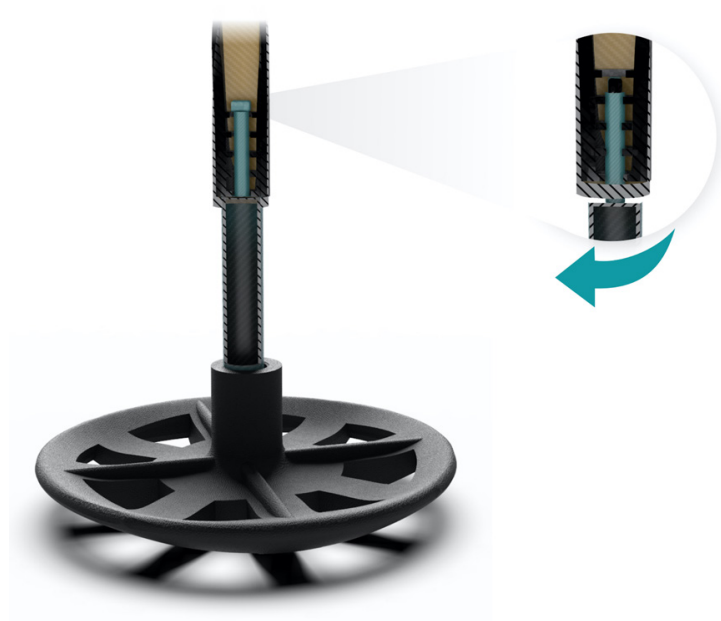

Fig. 13. Unión de contera regulable.

Por último, se muestra en la figura 14 una imagen de cómo se ve el nuevo diseño de las muletas Amphibio. Se puede notar que en su apariencia externa tiene pocas modificaciones, ya que los cambios implementados se encuentran, principalmente, a nivel de ensambles internos, como ya se explicó. 


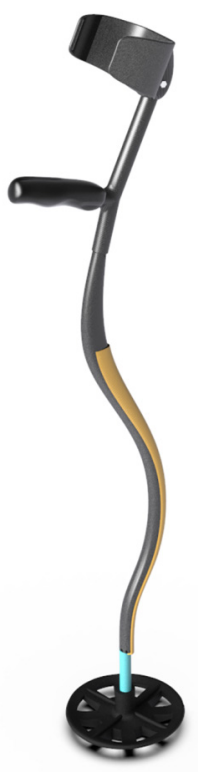

Fig. 14. Nuevo diseño de las muletas.

\section{Discusión}

Dados los cambios efectuados al diseño original, se realiza un análisis de los materiales. Se determinó el cambio de material a polietileno de alta densidad [13] con reforzamiento de fibra de vidrio; debido a las características que debe tener el producto, el material se seleccionó por medio del concepto de plásticos para la ingeniería [14], de este modo, se reduce el costo de producción, puede ser manufacturado a nivel nacional y el producto conserva las propiedades necesarias brindadas por los materiales para cumplir los requisitos establecidos. El material de la contera se mantiene siendo caucho natural [15] y se mantiene la silicona [16] como material por utilizar para el recubrimiento de la agarradera.

Al cambiar los materiales para manufacturar las muletas Amphibio, se cambiaron los procesos de manufactura. Los nuevos procesos se seleccionaron según la morfología de las piezas:

El moldeo por inyección [17] es utilizado para la fabricación de la abrazadera y el tubo de soporte de polietileno de alta densidad con reforzamiento de fibra de vidrio y el recubrimiento para la agarradera de silicona. Este proceso inicia con la colocación del molde, se plastifica el masterbatch, el cual es la mezcla de polímeros y aditivos, que se plastifican y son inyectados dentro del molde hasta ser llenado, donde adopta su forma al enfriarse.

El moldeo por inyección asistida por gas [18] es utilizado para manufacturar el tubo principal y el tubo regulable con polietileno de alta densidad con reforzamiento de fibra de vidrio, que poseen formas complejas. Este proceso se deriva del anterior, con la diferencia de ser inyectado parcialmente con el masterbatch plastificado y luego con gas, que comprime el material hacia las paredes formando piezas vaciadas. 
El moldeo por compresión con insertos [19] se selecciona para la fabricación de la contera donde se ensambla permanentemente con el tubo regulable como inserto. El proceso inicia, de igual forma, con la colocación del molde y en él se coloca la pieza como inserto; se introduce el caucho plastificado para que adopte la forma y se fije al inserto al enfriarse.

De este modo, se alcanza implementar las mejoras tanto en la fase de diseño del producto como en su manufactura, los cuales permiten que el producto pueda ser fabricado pensando en que su diseño sea óptimo dentro de la metodología de DFMA y, principalmente, logre el objetivo del Diseño para la Excelencia (DFX).

\section{Estrategia de manufactura}

Para comprender la estrategia planteada de manufactura, es necesario conocer las actividades presentes en todo el proceso del producto, detalladas con mayor profundidad, como se observa en la figura 15.

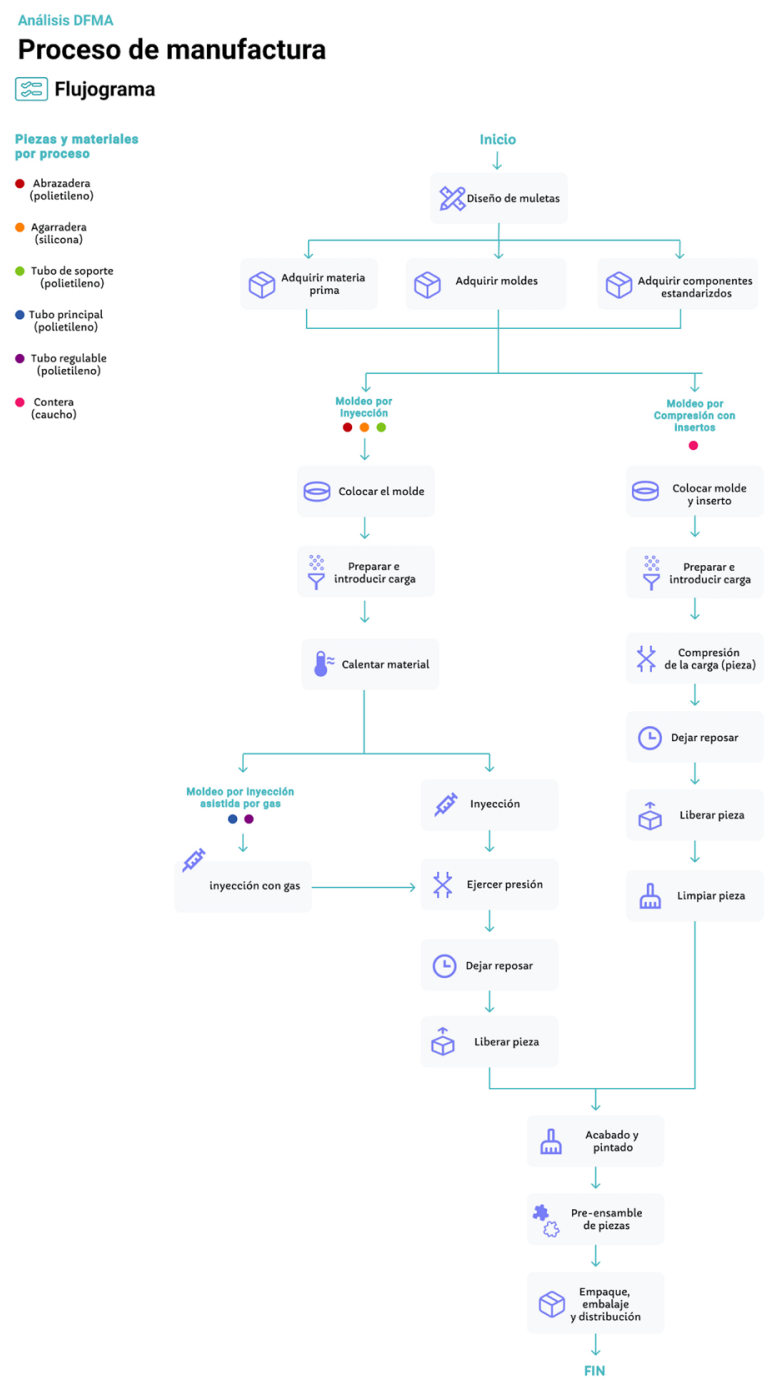

Fig. 15. Flujograma de la estrategia del proceso de manufactura para muletas Amphibio. 
La actividad inicial de diseño se concentra en definir el concepto, los requisitos, la morfología y los materiales que serán utilizados. De igual manera, se realizan modelos asistidos por computadora, donde se evalúa la distribución de fuerzas del diseño.

Posteriormente, se continúa con la fase de producción piloto, donde se realiza un prototipo para comprobar los tipos de uniones, dimensiones y el cumplimiento de los requisitos y funciones. Una vez se ha comprobado y corregido lo necesario, en caso de serlo, se procede a obtener las materias primas, moldes y componentes estandarizados para iniciar el proceso de manufactura. Los procesos de manufactura se realizan en su mayoría de manera simultánea, lo que permite agilizar la producción.

Al obtener todas las piezas manufacturadas, se procede al acabado y pintado. Luego se realiza un preensamble donde se coloca el recubrimiento de silicona de la agarradera y la abrazadera articulada, con el uso de un pasador, al tubo de soporte y este se une al tubo principal por medio de un encaje por presión.

Para finalizar, las muletas preensambladas junto con la contera regulable se embalan y son empacadas para proceder con su distribución a los puntos costeros y playas turísticas donde serán utilizadas. Siguiendo este planteamiento, se logra optimizar el proceso en cuanto recursos utilizados y el tiempo necesario para la producción de las muletas, como consecuencia de aplicar las metodologías analizadas del Diseño para la Excelencia.

Para potenciar las mejoras planteadas al producto, se proyecta que, en las investigaciones futuras, se pueda analizar con mayor profundidad el reciclaje de los materiales utilizados para su fabricación y su impacto ambiental. Asimismo, realizar un análisis exhaustivo de su ciclo de vida como producto, de modo que se comprenda las funciones que el ingeniero en diseño industrial tiene dentro de sus etapas de desarrollo. Otro factor importante por evaluar en el futuro es crear un producto piloto para generar pruebas con usuarios y asegurar su usabilidad y calidad.

\section{Conclusiones}

El diseño de un producto se centra en la necesidad del cliente, lo cual es imprescindible para satisfacer y cumplir las tareas requeridas. Sin embargo, el diseño debe contemplar más aspectos involucrados en todo el proceso, que un producto desde su inicio hasta su disposición final.

La metodología Design for X, empleada en el desarrollo de las muletas Amphibio, se rige por el concepto de analizar cada uno de los aspectos involucrados dentro del proceso de obtención del producto; para así concebirlo con los más altos estándares de calidad y usabilidad. Un aspecto que, por lo general, no es considerado por todos los ingenieros en diseño industrial y que ha mostrado alto impacto dentro de los resultados, es el proceso de manufactura. 
Esto se ve reflejado en el proceso de diseño, ya que es determinante para cumplir con los requerimientos planteados en una fase conceptual. Por esta razón, es necesario comprender que se debe crear un análisis que contemple los materiales, costos monetarios, tecnologías en procesos de manufactura, entre otras características, que, en conjunto, potencian el diseño, de modo que se logra optimizar el tiempo de producción e incluso los recursos utilizados.

Asimismo, al aplicar el método de DFMA dentro del diseño de un producto, se comprende cómo será ensamblado el producto y la dificultad que este puede presentar para los usuarios. Al aplicarse en el diseño de estas muletas en específico, se obtuvo como resultado una minimización de cantidad de piezas y partes que las conforman, sin alterar su cumplimiento con los requerimientos de diseño e incluso facilitando la usabilidad para los usuarios. Asimismo, se logró realizar un único ensamble que conserva la característica principal del producto, al poder modular su altura, lo que mejora las muletas en términos de usabilidad y en el proceso de producción.

Este proceso de diseño refleja la importancia de integrar todos los aspectos relacionados al producto, ya que, de este modo, garantiza los resultados, aparte de satisfacer a los usuarios. Esto genera múltiples beneficios para la empresa y considera el postuso del producto, de modo que también reduce las implicaciones ambientales que este podría tener. Así, la idea de que el diseño se realiza por un único departamento en la empresa está obsoleta, y todos los departamentos se involucran para brindar el producto con la mayor calidad, eficiencia y rentabilidad posible, que contemple cada aspecto de importancia, sin dejar de lado la satisfacción del cliente.

\section{Referencias}

[1] (INEC) Instituto Nacional de Estadística y Censos, "Primera Encuesta Nacional sobre Discapacidad 2018," May 2018 [Online]. Available: https://www.inec.cr/noticia/pais-tiene182-personas-en-situacion-de-discapacidad

[2] (INEC) Instituto Nacional de Estadística y Censos, "La población adulta mayor se triplicaría en los próximos 40 años" 2018.

[3] N. Palmer, "Design for Excellence (DFx) to Improve Product Cost, Quality, and Time to Market." [Online]. Available: https://blog.ttelectronics.com/design-for-excellence

[4] "Design for Manufacturing and Assembly (DFMA) | Siemens Software", Siemens Digital Industries Software, 2021. [Online]. Available: https://www.plm.automation.siemens.com/ global/es/our-story/glossary/design-for-manufacturing-and-assembly-dfma/53982.

[5] B. G.F and J. Carvalho, "Design for Manufacturing and Assembly methodology applied to aircrafts design and manufacturing," vol. 46, no. 7, pp. 116-121, 2013, doi: https:// doi.org/10.3182/20130522-3-BR-4036.00044. [Online]. Available: https://www. sciencedirect.com/science/article/pii/S1474667015356603 


\section{revistaIDI+}

[6] N. Palmer, "Design for Excellence (DFx) to Improve Product Cost, Quality, and Time to Market", Blog.ttelectronics.com, 2019. [Online]. Available: https://blog.ttelectronics.com/ design-for-excellence

[7] L. Asión Suñer, Estudio de los métodos de diseño modular y sus aplicaciones. 2017.

[8] J. Mule, "Design for Disassembly Approaches on Product Development", International Journal of Scientific \& Engineering Research, vol. 3, no. 6, 2012. Available: https://www. ijser.org/onlineResearchPaperViewer.aspx?Design-for-Disassembly-Approaches-onProduct-Development.pdf. [Accessed 28 June 2021].

[9] M. Ashby and K. Johnson, "Materials and Design: The Art and Science of - ProQuest", Proquest.com, 2014. [Online]. Available: https://www.proquest.com/legacydocview/ EBC/5754493?accountid=27651 [Accessed: 07- May- 2021]

[10] K. Soylu and J. C. Dumville, "Design for environment: The greening of product and supply chain," vol. 13, no. 1, pp. 29-43, 2011, doi: http://dx.doi.org/10.1057/mel.2010.19. [Online]. Available: https://www.proquest.com/scholarly-journals/design-environmentgreening-product-supply-chain/docview/852880942/se-2?accountid=27651

[11] "¿Qué es el diseño de servicios?", Ceupe.com, 2016. [Online]. Available: https://www. ceupe.com/blog/que-es-el-diseno-de-servicios.html [Accessed: 10- Jun- 2021].

[12] A. Taylor, "Design for Maintainability: Principles, Modularity and Rules", Design1st.com, 2014. [Online]. Available: https://www.design1st.com/Design-Resource-Library/design_ tips/Design_for_Maintainability.pdf.[Accessed: 28- Jun- 2021].

[13] "Polietileno de alta densidad", Tecnologiadelosplasticos.blogspot.com, 2021. [Online]. Available: https://tecnologiadelosplasticos.blogspot.com/2011/06/polietileno-de-altadensidad.html [Accessed: 28- Jun- 2021].

[14] P. Aceromafe, "Plásticos de ingeniería: descripción y usos | Aceromafe", Aceromafe, 2021. [Online]. Available: https://www.aceromafe.com/plasticos-de-ingenieriadescripcion-y-usos/ [Accessed: 28- Jun- 2021].

[15] "Conteras de caucho: todo lo que debes saber de tus muletas", Ayudatecnia.com, 2019. [Online]. Available: https://www.ayudatecnia.com/blog/conteras-de-caucho-todo-lo-quedebes-saber-de-tus-muletas [Accessed: 28- Jun- 2021].

[16] COATRESA, Coatresa.com, 2018. [Online]. Available: https://www.coatresa.com/ recubrimiento-de-silicona/ [Accessed: 10- Jun- 2021].

[17] "¿Qué es el moldeo por inyección? | Moldeo por inyección | Introducción al Maquinado | KEYENCE México", Keyence.com.mx, 2017. [Online]. Available: https://www.keyence. com.mx/ss/products/measure-sys/machining/injection-molding/about.jsp [Accessed: 10Jun- 2021].

[18] V. perfil, "Moldeo por inyección asistida con gas (GAIM)", Tecnologiadelosplasticos. blogspot.com, 2021. [Online]. Available: https://tecnologiadelosplasticos.blogspot. com/2012/05/moldeo-por-inyeccion-asistida-con-gas.html [Accessed: 10- Jun- 2021]. 


\section{revistaIDI+}

[19] "Moldeo por compresión", Tecnologiadelosplasticos.blogspot.com, 2021. [Online]. Available: https://tecnologiadelosplasticos.blogspot.com/2011/10/moldeo-porcompresion.html [Accessed: 10- Jun- 2021]. 